\title{
GENETIC ALGORITHMS FOR GNC SETTINGS AND DACS DESIGN APPLICATION TO AN ASTEROID KINETIC IMPACTOR
}

\section{P. Vernis and V. Oliviero}

ARIANE Group

Les Mureaux, France

This paper deals with an application of Genetic Algorithm (GA) tools in order to perform and optimize the settings phase of the Guidance, Navigation, and Control (GNC) data set for the endgame phase of a Kinetic Impactor (KI) targeting a medium-size Near Earth Object (NEO). A coupled optimization of the GNC settings and of the GC-oriented design of the Divert and Attitude Control System (DACS) is also proposed. The illustration of the developed principles is made considering the NEOShield study frame.

\section{ACRONYMS AND NOTATIONS}

$\begin{array}{ll}\text { ABM } & \text { Antiballistic Missile } \\ \text { ACS } & \text { Attitude Control System } \\ \text { AOCS } & \text { Attitude and Orbit Control System } \\ \text { ATV } & \text { Automated Transfer Vehicle } \\ \text { CoG } & \text { Center of Gravity } \\ \text { DACS } & \text { Divert and Attitude Control System } \\ \text { DG } & \text { Differential Games } \\ \text { DoF } & \text { Degree-of-Freedom } \\ \text { EKF } & \text { Extended Kalman Filter } \\ F_{\text {ACS }} & \text { ACS thrust level } \\ F_{\text {Divert }} & \text { Divert thrust level } \\ \text { FoV } & \text { Field-of-View } \\ \text { GA } & \text { Genetic Algorithms } \\ \text { GNC } & \text { Guidance, Navigation, and Control } \\ \text { HF } & \text { High Frequency } \\ \text { HOMER } & \text { HOver ManeuvER } \\ \text { IMU } & \text { Inertial Measurement Unit } \\ \text { KI } & \text { Kinetic Impactor } \\ \text { LORRI } & \text { LOng Range Reconnaissance Imager }\end{array}$

(C) The Authors, published by EDP Sciences. This is an open access article distributed under the terms of the Creative Commons Attribution License 4.0 (http://creativecommons.org/licenses/by/4.0/). 


$\begin{array}{ll}\text { LoS } & \text { Line-of-Sight } \\ M & \text { Miss-distance } \\ \text { MIB } & \text { Minimum Impulse Bit } \\ \text { MPS } & \text { Main Propulsion System } \\ \text { MTCL } & \text { MonTe-CarLo } \\ \text { NEO } & \text { Near Earth Object } \\ \text { SIRU } & \text { Space Inertial Reference Unit } \\ T & \text { Time-to-go } \\ V & \text { Closing velocity } \\ \text { YPR } & \text { Yaw, Pitch, and Roll } \\ \Delta T_{\text {ACS }} & \text { ACS call/firing period } \\ \Delta T_{\text {Divert }} & \text { Divert call/firing period }\end{array}$

\section{INTRODUCTION}

The collision probability with the up-to-now listed NEOs is assessed to be small, but not null, and the impact with a small to medium-class asteroid (50 to $100 \mathrm{~m}$ in diameter) could be catastrophic. Even if such event is not highly likely in a near future, preparing the technologies aiming at mitigating such event appears obvious when looking back, for example, at the damages generated by a 20 -meter diameter meteor that exploded over Chelyabinsk.

Within the frame of the NEOShield activities of the 7th Framework Programme for Research and Technological Development (FP7) funded by the European Council, a consortium of scientific and industrial partners has been set to assess different mitigation techniques: destruction by a surface or an underground nuclear blast or deflection using ion-beam propulsion systems, gravity, or solar sail tractors but also kinetic impactor. Among these different techniques, and as more or less already experienced during the Deep Impact mission (headon collision with the Tempel 1 comet), the most promising one seems to be the kinetic impactor.

The main reasons for choosing this solution are, first, the lower complexity of the intercept/impact mission compared to the other solutions and, then, the possible reuse of technologies developed within the frame of missile defence program. By comparison with a missile defense intercept mission, the mission requirements are relaxed with a final miss-range between 25 and $50 \mathrm{~m}$. But the stress is put on the Navigation side: a long range (NEO acquisition may be done up to $24 \mathrm{~h}$ before impact) and very narrow field-of-view (FoV) visible camera (only $\pm 0.3^{\circ}$ ) is used to acquire and track the NEO until impact. Refurbishing existing GNC solutions that were extensively assessed during past exoatmospheric intercept analyses, 6 degree-of-freedom (DoF) Monte-Carlo (MTCL) simulations performed on a set of 4 endgame missions (from very optimistic to very pessimistic kinematic conditions at endgame handover: 30- up to 600-kilometer 
initial miss-distance) and for two classes of liquid-fuelled DACS (200- or 3500newton divert) produced interesting results almost fully in line with the mission requirements for most of the cases [1]. The most tricky and time-consuming task to get such GNC performance level was to tune all the GNC data: Guidance and Control internal data and especially the 8-state Extended Kalman Filter (EKF) parameters, the EKF objective being to estimate the miss-distance and the timeto-go using only line-of-sight (LoS) angle measurements whereas miss-distance and time-to-go become well observable only very late.

In order to ease the GNC setting phase (up to 15 parameters have to be set) but also to optimize, if needed, the DACS definition (5 additional tuning parameters), a dedicated process relying on GA has been implemented. Considering as reference baseline the MTCL results obtained during the NEOShield activities, this paper presents the impact on the GNC performance of a GA setting of the GNC chain for a fixed DACS design (medium or high thrust) and of a coupled GNC setting and DACS design optimization over the same set of endgame missions.

The first part of this paper is dedicated to a quick survey of the NEOShield framework and of the GNC chain retained for the medium to high thrust DACS kinetic impactor and its performance. Next, the GNC performance as obtained at the end of the NEOShield GNC activities is presented. Then, the GA-based process enabling an autonomous and robust setting of the GNC data and, if selected, a global optimization of the GNC data set and of the DACS main features is presented, and a comparison between "handmade" and GA settings is proposed relying on 6 DoF MTCL simulation results.

\section{NEOShield FRAMEWORK}

One of the objectives of the NEOShield program was to investigate different NEO mitigation solutions. Among them, KI appeared to be the most realistic one because of the potential heritage of exoatmospheric missile defence activities. Namely, the major differences between missile defence and planetary defence endgame tasks that are given to the interceptor/impactor are essentially a matter of scale factor:

- the acquisition by the onboard sensor of a NEO can be made up to $24 \mathrm{~h}$ before impact but no more than a few hundreds of seconds for an incoming warhead;

- the expected final miss-range lays in the range of 25 to $50 \mathrm{~m}$ for a mediumsize NEO, but only a few tens of centimeters for a warhead (direct hit objective). For a missile defence kill vehicle, the objective of the endgame phase is to destroy its target, whereas for a KI, it is only necessary to push 
it, the kinetic energy transfer acting directly on the orbital parameters of the NEO interplanetary path. Considering that the NEO impact occurs millions of kilometers away from Earth, even a very small $\Delta V$ induced by the KI can be enough to deviate the NEO's trajectory away from Earth;

- the initial miss-distance at endgame handover can be very large for a NEO, a few tens of kilometers, whereas it is much less for a missile defence interceptor. But because of the extended endgame duration, such extra initial miss-distance can easily be managed; and

- the closing velocity on a NEO can be very high, up to several tens of kilometers per second for a head-on collision. But if we limit the engagement scenarii to only pursuit the lateral configuration with prograde collision route, it is possible to keep the KI-NEO closing velocity $(11.6 \mathrm{~km} / \mathrm{s}$ for the retained scenario) to values similar to some antiballistic missile (ABM) configurations. Figure 1 summarizes the main engagement conditions considered within the frame of the NEOShield activities.

Figure 1 illustrates a typical impact mission on a threatening asteroid.

Doing so, reusing already investigated GNC algorithmic solutions as well as propulsive architecture (a liquid-fuelled DACS is the baseline) can be considered to assess the feasibility of the NEO impact mission. Because of the very long range NEO acquisition by the onboard camera, there is no real need for a powerful divert system as it could be required for an exoatmospheric interceptor. Within the NEOShield framework, three DACS concepts have been considered relying on a 20- (satellite-like attitude and orbit control system (AOCS) engine), a 200- (ACS engine from the automated transfer vehicle (ATV), Fig. 2a), and

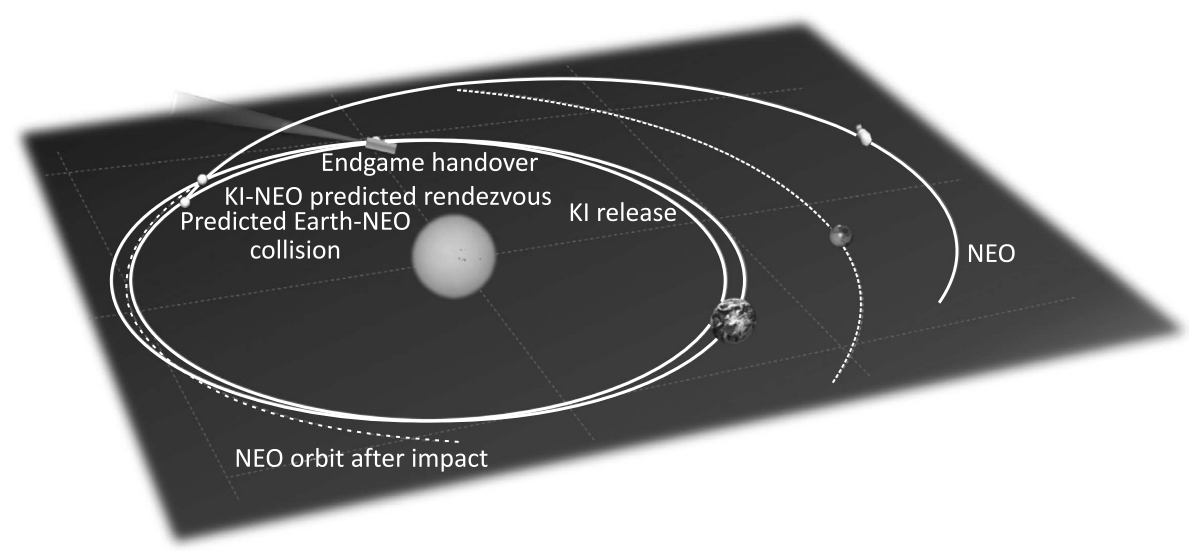

Figure 1 Near Earth object mitigation using a KI 


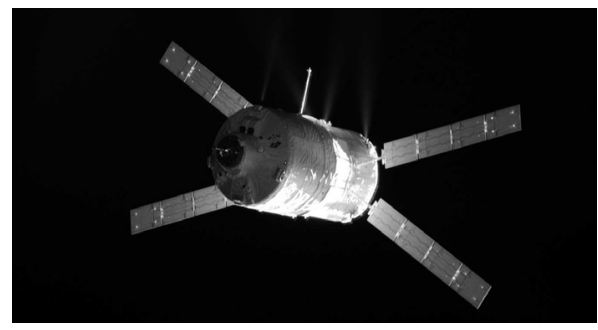

(a)

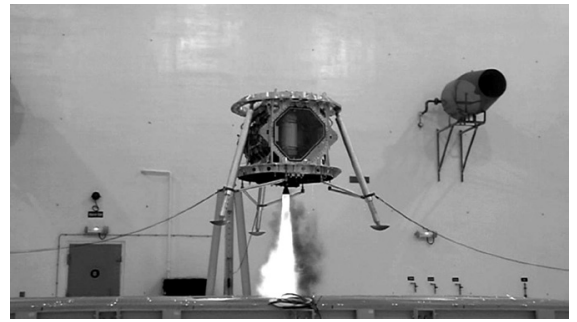

(b)

Figure 2 The ATV ACS (a) AND HOMER MPS ( $b$ ) firings

Table 1 The DACS design

\begin{tabular}{lccc}
\hline \multicolumn{1}{c}{ Engine } & Medium thrust KI, & High thrust KI, & $\begin{array}{c}\text { Firing period, } \\
\text { ms }\end{array}$ \\
& $\mathrm{N}$ & $\mathrm{N}$ & 2500 \\
\hline Divert & 200 & 175 & 50 \\
ACS (yaw and pitch axes) & 20 & 90 & 50 \\
ACS (roll axis) & 10 & & \\
\hline
\end{tabular}

a 3500-newton divert (main propulsion system (MPS) engine from the Hover Maneuver (HOMER) test platform [2], Fig. 2b).

This paper concerns only the medium $(200 \mathrm{~N})$ to high thrust $(3500 \mathrm{~N})$ level divert solutions that allow a reduced endgame phase. The nominal data of the DACS are as presented in Table 1.

With such propulsive characteristics, the endgame phase can be entered much later than with the 20-newton satellite-like solution leaving more time for observing the asteroid and increasing the performance of the aimed point selection phase (Fig. 3) for the corresponding GNC timeline.

Within the frame of the NEOShield GNC activities, only the endgame phase is simulated (dedicated 6 DoF simulation tool). Thus, for simulation purpose, in order to ease the convergence of the Navigation filter for estimating the initial miss-distance, an observation phase (minimum $50 \mathrm{~s}$ ) is added to the previous timeline at the beginning of the endgame phase. During this preliminary phase, only KI attitude corrections are performed.

The main point with respect to the design objective is to locate the nominal center-of-gravity (CoG) of the vehicle centered in the Divert positioning plane (4 thrusters aligned on yaw and pitch axes) in order to limit disturbing torques that would be generated by the divert firings with CoG off-centering and/or thrusters misalignments. The ACS which is located at the back of the vehicle is designed with 4 thrusters for the pitch and yaw control, and a set of 4 others 


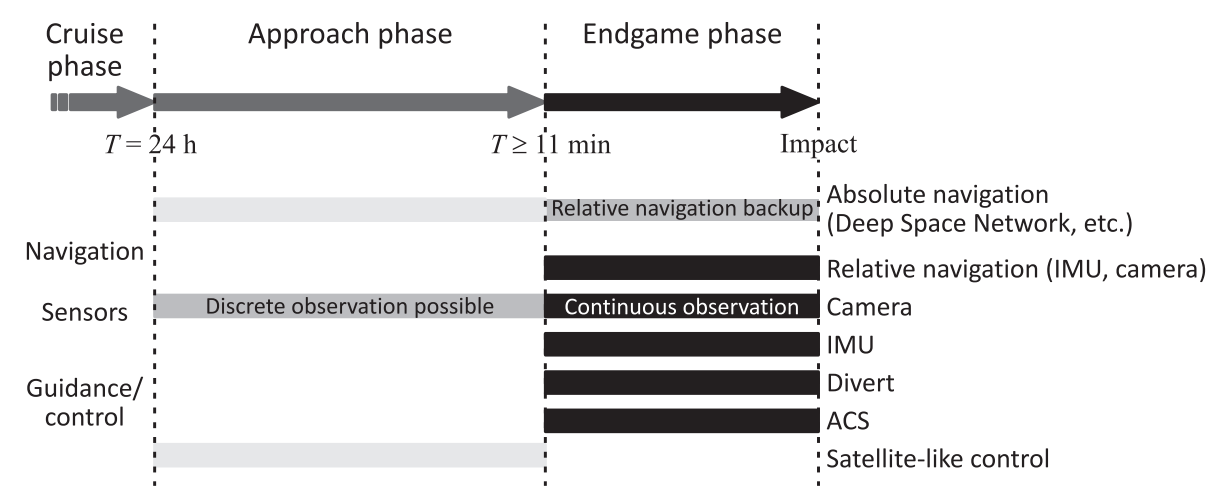

Figure 3 GNC timeline, medium to high thrust diver KI

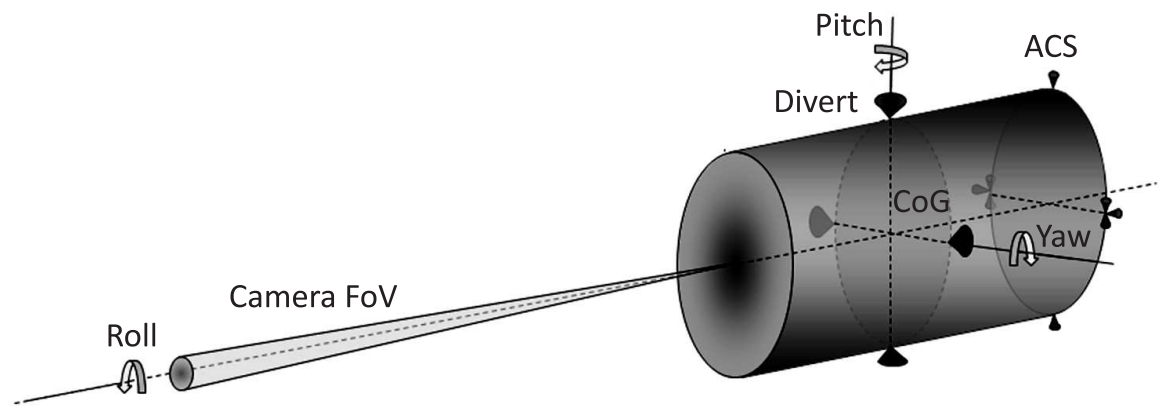

Figure 4 Kinetic impactor simplified model

smaller thrusters for the roll control (see Fig. 4 displaying a very schematic view of the KI design).

Table 2 summarizes the main features of the KI (550-kilogram class vehicle).

The main onboard sensors are a LORRI-type narrow angle visible camera $( \pm 0.38$ degree FoV) and a space inertial reference unit (SIRU) from Northrop Grumman.

Table 2 Kinetic impactor main features

\begin{tabular}{cccc}
\hline $\begin{array}{c}\text { Spacecraft } \\
\text { mass, } \mathrm{kg}\end{array}$ & Spacecraft size & $\begin{array}{c}\text { Spacecraft } \\
\text { inertia, } \mathrm{kg} \cdot \mathrm{m}^{2}\end{array}$ & CoG excursion \\
\hline 550 & $\begin{array}{c}\text { Cylinder of 3.8-meter } \\
\text { height and 1.97-meter } \\
\text { diameter }\end{array}$ & $\begin{array}{c}2140 \text { (yaw } \\
\text { and pitch axes) }\end{array}$ & $\begin{array}{c}\text { Longitudinal deviation - } \\
\text { max } \pm 10 \mathrm{~cm}\end{array}$ \\
$\begin{array}{c}\text { Transverse deviation }- \\
\text { max } \pm 5 \mathrm{~cm}\end{array}$ \\
\hline
\end{tabular}


For the medium to high thrust divert KI, the main features of the endgame mission to perform are defined by the time-to-go $T$, or time remaining before impact, and the miss-distance $M$, or predicted final miss-range with no further KI trajectory correction, from very optimistic (scenario 1) to very pes-

Table 3 Endgame scenarii

\begin{tabular}{ccc}
\hline Scenario & $\begin{array}{c}M_{0} \text { maximum } \\
(3 \sigma), \mathrm{km}\end{array}$ & $\begin{array}{c}T_{0} \text { nominal, } \\
\mathrm{s}\end{array}$ \\
\hline 1 & 30 & 700 \\
2 & 90 & 1500 \\
3 & 200 & 3300 \\
4 & 600 & 10300 \\
\hline
\end{tabular}
simistic (scenario 4) conditions at endgame handover (Table 3).

The baselined targeted final miss-range is $50 \mathrm{~m}$ (99.7\% value) but for smaller NEO, a 25-meter final miss-range might be required (secondary objective).

\section{GNC CHAIN FOR THE ENDGAME PHASE OF A KINETIC IMPACTOR}

For a kinetic impactor, the Guidance, Control, and Navigation objectives are as listed hereafter:

- the Guidance is in charge of cancelling out the miss-distance, i. e., the predicted final miss-range, and to ensure that it will be null, or below the mission requirement, at the time of impact;

- the Control is in charge of pointing the strapdown camera onto the target and keeping the image of the target centered on the seeker focal plane. A secondary but essential objective is also to limit the dynamic disturbances (angular shifts and angular rates increases) induced by the divert firings. A third objective is eventually to limit the gyroscopic torques and to keep the roll rate as low as possible; and

- last but not least because it will be the main driver for the GNC performance, the Navigation is in charge of feeding the Guidance and Control functions with accurate estimates of the miss-distance $M$ and the time-togo $T$ (Guidance), and the LoS angles, LoS and yaw, pitch, and roll (YPR) rates (Control). Within the frame of this study, we will rely on a simplified performance model of the sensor: point-mass model for the target, no subpixel processing, and approximately a 1-second terminal blinding phase.

Within the frame of missile defence activities, the endgame guidance has been extensively investigated, from the most classic proportional navigation scheme [3] 
and its different forms $[4,5]$, to most recent development relying on differential games (DG) $[6,7]$. More recently, further analyses have been carried out for planetary defence objectives $[8,9]$. Nevertheless, in order to benefit from past activities carried out at AIRBUS Defence and Space and considering that the impact angle was not a severe constraint and mainly obtained at endgame handover, in-house GNC solutions have been reused with only light adaptations.

Without getting into the details of the GNC algorithms, the retained Guidance scheme relies on DG theory (even if the target is not maneuvering, the miss-distance estimation errors or the divert discrepancies can be regarded as pseudomaneuvering capabilities of the target). Such guidance algorithm that defines in flight autonomously the divert firings is well suited to manage the final accuracy (to get $M=0 \mathrm{~m}$ at $T=0 \mathrm{~s}$ ), the fuel budget, and the performance of the Navigation function by building command barriers in the $(M, T)$ space, splitting the $(M, T)$ space in predefined command zones. Figure 5 illustrates the behavior of the DG guidance algorithm (scenario 1). The initial 50-second observation time period is needed for the convergence of the relative navigation filter, the approach being not simulated.

The main setting parameters of the guidance are mainly linked to the consumption barrier that is added to the command barriers when an indetermination occurs in the choice of the command to apply (a pulsed-mode divert has been used). And a stop criterion of the guidance is added in order to limit potential degradation on the miss-distance due to a slow and late convergence of the timeto-go in parallel of a quick divergence of the LoS rate due to the KI-NEO closing at the very end of the endgame phase. The consumption barrier is modeled by a scaling factor $k_{G}$ ranging from 0 to 1 that is linearly time-to-go depen-

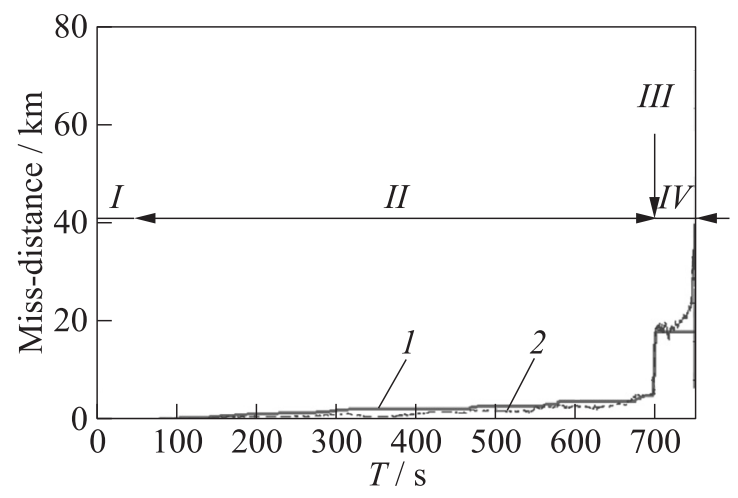

Figure 5 Differential games guidance operating mode: 1 - real value; 2 - estimated value; $I$ - final cancellation (final occuracy); $I I$ - intermediate adjustments (fuel budget management); III - initial cancellation (capture); and $I V-50$-second observation 


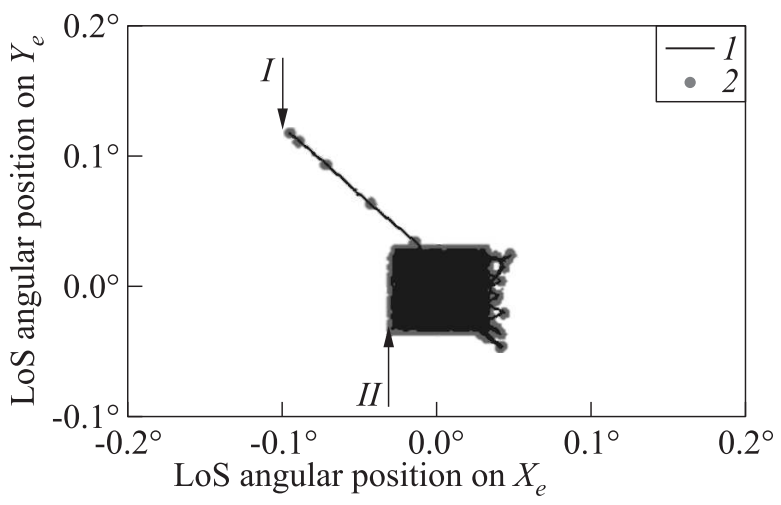

Figure 6 Minimum time with limit cycle Control operating mode (with fixed tracking zone): 1 - simulated value; 2 - observed value; $I$ - NEO at endgame handover; and $I I- \pm 0.3$ degree tracking zone

dent from $T_{0}$ till $T_{0}-\Delta T_{G}$ and then constant. The stop criterion is defined by a time-to-go $\left(T_{\text {off }}\right)$ and/or a miss-distance $\left(M_{\text {off }}\right)$ condition.

The Control is decoupled into a minimum time command with limit cycle on the pitch and yaw axes for the LoS control and a simple bang-bang regulation of the roll rate for limiting the gyroscopic torques. Figure 6 illustrates the behavior of the pitch and yaw control algorithm, with a tracking zone limited to $\pm 0.03^{\circ}$.

The Control settings parameters are directly linked to the angular size of the limit cycle that defines the tracking submatrix inside the seeker focal plane and the maximum roll rate triggering roll firings. As for the guidance, and mainly to limit the ACS budget, the size of the tracking zone is assumed linearly decreasing from endgame handover till $T_{0}-\Delta T_{C}$, and then constant beyond.

Eventually, the Navigation objective is mainly to estimate the miss-distance and the time-to-go using only LoS angle measurements [10] via the visible camera. An absolute Navigation relying only on the IMU (inertial measurement unit) data is always active and a relative Navigation using both IMU and camera is enabled once the target has been locked on, ground stations telemetry data being used only to perform Trajectory Correction Maneuvers before being in sight of the asteroid (up to $24 \mathrm{~h}$ before impact). Thus, once the endgame phase is entered, the KI is fully autonomous and it uses only the relative navigation outputs, the absolute navigation which is still running being just a backup solution. The relative Navigation relies on an 8-state extended Kalman filter (LoS and LoS rate components, YPR rates, and time-to-go), LoS components and YPR rates being measured. Figure 7 illustrates the behavior of the EKF with regard to the most important parameters that are given by the LoS rates components and the time-to-go which are directly used to evaluate the miss-distance under 


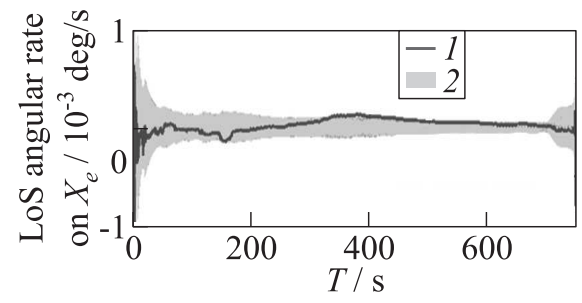

(a)

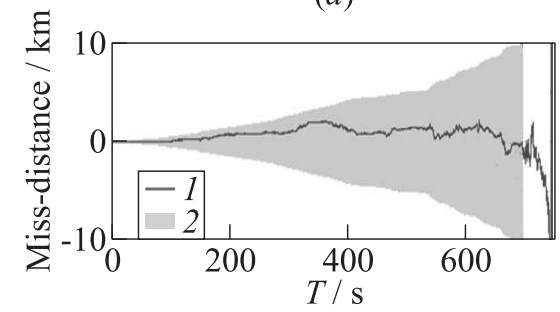

(c)

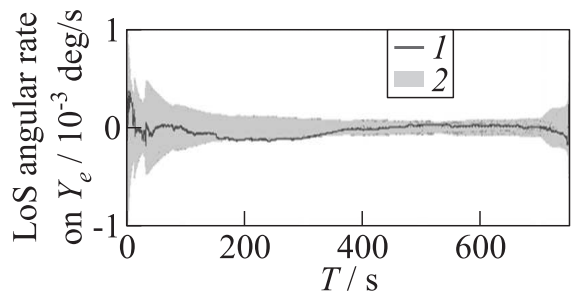

(b)

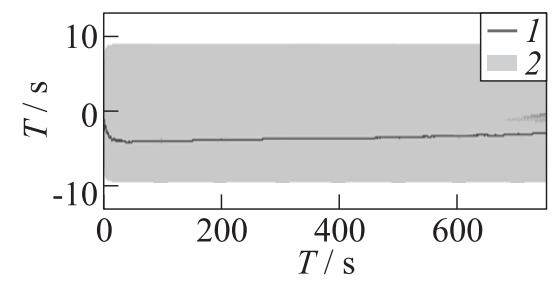

(d)

Figure 7 The EKF vision-based relative navigation operating mode: (a) LoS angular rate on $X_{e} ;(b)$ LoS angular rate on $Y_{e} ;(c)$ miss-distance; $(d)$ time-to-go; 1 estimation performance; and $2-3 \sigma$ confidence level

the simplifying assumption of a constant closing velocity. When the EKF is well tuned, the terminal divergence that can be observed on the LoS rates in the last 100 to $50 \mathrm{~s}$ of the endgame phase is normally compensated by the convergence of the time-to-go which occurs only late due to the difficulty to estimate a radial distance (the time-to-go) using only transverse distance measurements (the LoS angle components).

Because the performance of the relative Navigation will directly drive the GNC performance, its tuning is the trickiest part of the GNC activity, once the GNC algorithms have been designed, implemented, and unitary validated. The set of settings parameters is also much larger than for the Guidance or the Control because it considers the initial value of the covariance matrix and the modeling noise standard deviations arising from the linearization and discretization steps of the state model (camera and IMU measurements are discrete whereas the KI-NEO state model is continuous).

\section{GNC PERFORMANCE WITH CLASSIC HANDMADE GNC TUNING}

First, the feasibility of the impact mission was demonstrated in the optimal but nonrealistic case of an ideal Navigation function (the KI-NEO state vector is 

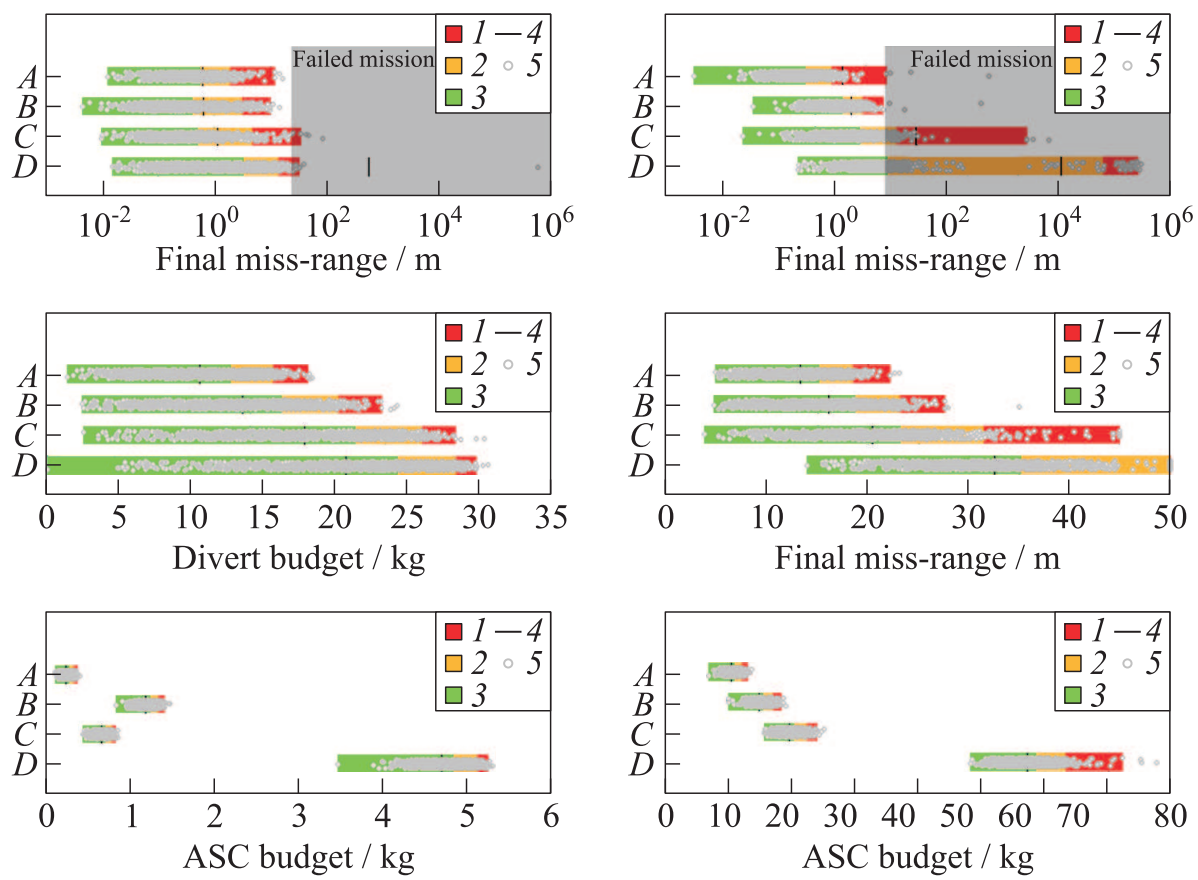

Figure 8 The GNC performance, handmade GNC settings: $A-$ scenario $1,30 \mathrm{~km}$; $B$ - scenario $2,90 \mathrm{~km}$; $C$ - scenario $3,200 \mathrm{~km} ; D$ - scenario $4,600 \mathrm{~km}$; left column - 200-newton divert; right column - 3500-newton divert; $1-99.7 \%$ or $3 \sigma$ domain; $2-95.4 \%$ or $2 \sigma$ domain; $3-68.2 \%$ or $1 \sigma$ domain; 4 - arithmetic mean value; and 5 - MTCL runs

estimated without any error) with a 100 percent hit probability and for a final miss-range always below $3.5 \mathrm{~m}$. Next, the GNC setting phase has been refurbished to handle the discrepancies introduced by the real performance of the relative navigation. After many trial-and-errors settings with a continuous increase of the performance, the settings process has been stopped to yield the GNC performance as summarized by Fig. 8 .

Table 4 summarizes the corresponding hit probabilities for a 50- and a 25 meter final miss-range objective.

The mission requirement expressed towards the so-termed $3 \sigma 50$-meter hit probability is almost met for the 200-newton divert case whatever are the initial endgame conditions (a longer investment in the tuning phase should yield such result: there is no reason for having a 99.8 percent success rate with the scenario 4 but only $99.5 \%$ with the scenario 2 which is less stressing). Such success rate is degraded for the $3500 \mathrm{~N}$ KI configuration, especially on scenario 4 for which the image of the target is lost shortly after the first divert firings due to a too weak 
Table 4 Monte-Carlo results

\begin{tabular}{crrrr}
\hline \multirow{2}{*}{ Scenario } & \multicolumn{2}{c}{ Phit $50 \mathrm{~m}$} & \multicolumn{2}{c}{ Phit $25 \mathrm{~m}$} \\
\cline { 2 - 5 } & \multicolumn{1}{c}{$200 \mathrm{~N}$} & $3500 \mathrm{~N}$ & \multicolumn{1}{c}{$200 \mathrm{~N}$} & $3500 \mathrm{~N}$ \\
\hline 1 & $100.0 \%$ & $99.8 \%$ & $100.0 \%$ & $99.7 \%$ \\
2 & $100.0 \%$ & $99.8 \%$ & $100.0 \%$ & $99.7 \%$ \\
3 & $99.9 \%$ & $97.5 \%$ & $99.6 \%$ & $92.3 \%$ \\
4 & $99.9 \%$ & $86.1 \%$ & $99.3 \%$ & $67.0 \%$ \\
\hline
\end{tabular}

ACS, the ACS firing period (50 ms) being constrained by the CPU time of the image processing (sizing hypothesis). In those cases, the Guidance lives only on the prediction of the target motion yielding in most of the simulated cases a final miss-distance well over the initial one.

The secondary mission objective (25-meter hit probability at $3 \sigma$ ) is also almost fulfilled for the 200-newton case what is far to be true for the 3500-newton case whose mean 25-meter hit probability is around $97 \%$, except for the scenario 4 for which it drops down to less than $70 \%$.

Nevertheless, because of the continuous improvement of the GNC performance and even if the main mission requirement is not fully met, the obtained results with a handmade but also time-limited GNC settings process opened the way to a potential improvement of the performance through an optimization of the GNC data set.

\section{GNC SETTINGS WITH GENETIC ALGORITHMS}

In order to shorten the settings phase process but also to try to optimize it and to enhance the GNC performance, an automated process has to be implemented. According to some previous experience in that domain for roughly similar problems [11], the retained optimization process is based on GA. Indeed, it may be a good candidate to solve such a complex problem as long as the optimization problem has been well set and the optimization criterion well defined. For the current application, the DACS propulsive design (i. e., thrust level and firing period) is frozen, even if the ACS appears slightly undersized for the 3500-newton divert configuration as explained above. The optimization problem to solve is then to find the GNC data set (15 parameters to tune: 3 for the Guidance, 4 for the Control, and 8 for the Navigation as presented in section 3) enabling the fulfilment of the mission requirement expressed towards the hit probability for the 4 scenarii and per KI configuration. Because of the various conditions at endgame handover, the optimization of the GNC data will be performed scenario per scenario at this stage of the project. If improving the performance goes naturally through the increase of the hit probability, one will try to minimize 
the fuel budget that was initially not constrained in the previous part of the study. This additional constraint helps in differentiating potential solutions for the Guidance and Control data set, the objective of the time evolution of the scaling factor $k_{G}$ as well as the size of the tracking submatrix aiming to limit the divert and ACS fuel budgets.

The performance of the Guidance is directly linked with the divert fuel budget and the final miss-range at impact.

The performance of the Control that will partially drive the performance of the Guidance is defined by the limitation of the fuel budget and the ability to keep the image of the target well inside the tracking submatrix all along the endgame phase. Some transient excursions out of this tracking sub-matrix are enabled as long as the Control is able to put it back inside this reduced FoV. But defining a performance index for the Navigation is less obvious as it is the whole behavior of the navigation filter all along the endgame phase that will drive the performance of the GNC chain: terminal values may be well estimated with only a few errors, if the so-termed $3 \sigma$ confidence interval has been violated too many times before impact, Guidance and Control will have fired too often the DACS thrusters yielding a too large fuel consumption and possibly a poor final miss-range. The performance of the Navigation function is thus modeled via the offset between the estimated error and the $3 \sigma$ confidence interval barrier (upper or lower barrier according to the sign of the estimated error).

In order to make it as simple as possible, the objective function to be minimized by the GA process has been defined, after many trials, as an algebraic sum of elementary weighted performance indices $c_{i}$ as

$$
F_{\text {obj }}=\sum_{i=1}^{n} w_{i} c_{i}
$$

with the elementary cost functions $c_{i}$ as depicted in Table 5 and ranked by order of importance.

For illustration purpose, the objective function retained in the end for scenario 1 is the following one, all bolded printed values highlighting the parameters/weights that are modified from one scenario to the other one:

$$
\begin{aligned}
F_{\text {obj }}=1000 c_{1 \text { mean }}+\mathbf{2 0} c_{1 \text { max }} & +\left(100-c_{2}\right)^{3}+\mathbf{2 0} c_{3}+\mathbf{1 5 0} c_{4} \\
+ & \text { 100abs }\left(c_{5}\right)+\frac{c_{6}}{\mathbf{3}}+\left(\frac{c_{7}}{\mathbf{3}}\right)^{\mathbf{3}}+500 c_{8}+500 c_{9} .
\end{aligned}
$$

For the performance index linked to the miss-distance, the mean value of the final miss-range $\left(c_{1 \text { mean }}\right)$ as well as the maximum one $\left(c_{1 \max }\right)$ have been considered.

In addition to tuning the GA process (number of individuals, epochs, selection, crossover, mutation and reinsertion processes, etc.), one of the difficulties 
Table 5 Elementary cost function list

\begin{tabular}{cl}
\hline $\begin{array}{c}\text { Cost } \\
\text { function }\end{array}$ & \multicolumn{1}{c}{ Criterion } \\
\hline$c_{1}$ & Final miss-range \\
\hline$c_{2}$ & Success rate (i. e., 50-meter hit probability) \\
\hline$c_{3}$ & Divert fuel budget \\
\hline$c_{4}$ & ACS fuel budget \\
\hline$c_{5}$ & Estimated final time-to-go \\
\hline$c_{6}$ & Miss-distance estimation error at the end of the initial convergence phase \\
\hline$c_{7}$ & Number of NEO images lost by the sensor (over the endgame phase) \\
\hline$c_{8}$ & $\begin{array}{l}\text { Time-to-go estimation error (from end of initial convergence phase till } \\
\text { impact) }\end{array}$ \\
\hline$c_{9}$ & $\begin{array}{l}\text { Miss-distance estimation error (from end of initial convergence phase till } \\
\text { impact) }\end{array}$ \\
\hline
\end{tabular}

Table 6 The GA settings

\begin{tabular}{ll}
\hline \multicolumn{1}{c}{ GA step } & \multicolumn{1}{c}{ Method } \\
\hline Selection & Stochastic universal sampling \\
Crossover & Intermediate recombination (80 percent recombination rate) \\
Mutation & Breeders-like real-valued mutation (5 percent mutation rate) \\
Reinsertion & Global reinsertion (70 percent insertion rate) \\
Migration & Not considered \\
\hline
\end{tabular}

of using GA is to define the objective function and the associated weights. For instance, the settings of the objective function for scenario 1 or scenario 4 are very different.

All the results presented hereafter have been obtained considering the GA tuning shown in Table 6 .

The number of epochs has been fixed at 50 (no convergence criterion has been considered to stop the GA process) for 50 individuals with 4 chromosomes $(G$, $N$, and $C$ settings and GNC performance) for a total of 15 genes per individual. The objective function is evaluated for each individual over a limited MTCL process including 50 runs each, with every time a new drawing of the dispersions and uncertainties. It has to be noted that migration between subpopulations has been investigated but without any real conclusive results. In the end, it has been discarded from the GA baseline.

Figure 9 depicts the implementation of the GA process and the interaction with the 6 DoF SpaceShield simulation tool (dark grey boxes when used).

Because the step before the GA process implementation was a handmade trial-and-error setting of the GNC data, the results obtained during that first 


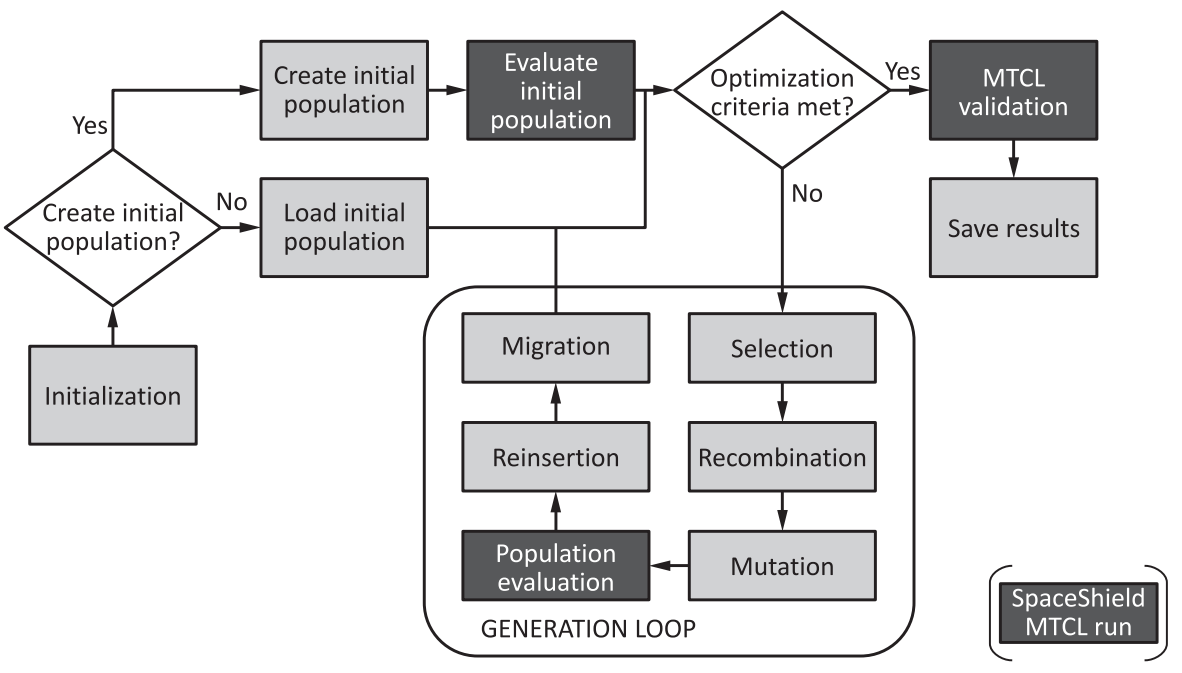

Figure 9 SpaceShield simulation tool and GA implementation

step were loaded as initial population, but a pure random initial population could have been considered.

Unfortunately and mainly for a simple question of computation time (not less than 7-day computation for the GA process applied to scenario 4, for a single optimization of the GNC data), the optimization of the GNC data for the scenario 4 has been stopped because of a poor convergence process (see comparison of the time history of the cost function for scenarios 1 and 4 in Fig. 10) and no significant improvement of the GNC performance obtained with a trial-and-error handmade setting. For illustration purpose, Fig. 10 presents the evolution of the fitness per individual and per epoch for the scenarii 1 and 4, with the same GA settings except the objective function.

One can observe a rather quick convergence, only 10 epochs being enough to get the convergence for scenario 1 whereas 50 epochs are clearly too few for scenario 4 with very different terminal values per individual.

Scenario 4 being the most pessimistic one and the less probable one, it was decided to focus only on the three first scenarios.

The radar plots (Fig. 11) display a comparison between the initial handmade and the optimized GA setting of the GNC data for the 200- and 3500-newton divert cases (the smallest the surface, the better performance). The retained 5 comparison criteria are:

(1) the 99.7 percent (or $3 \sigma$ value) final miss-range;

(2) the divert fuel budget; 


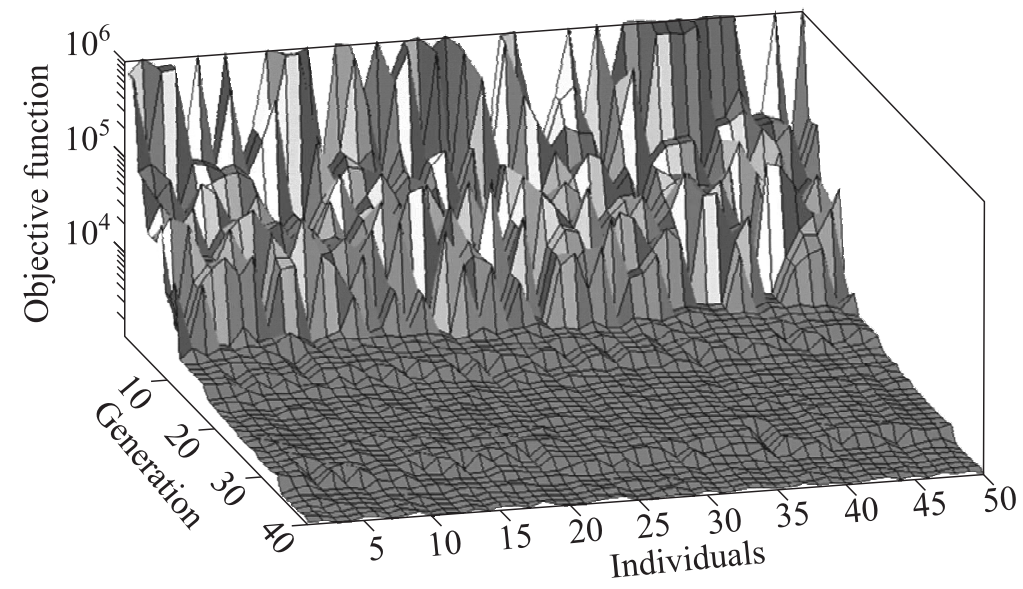

(a)

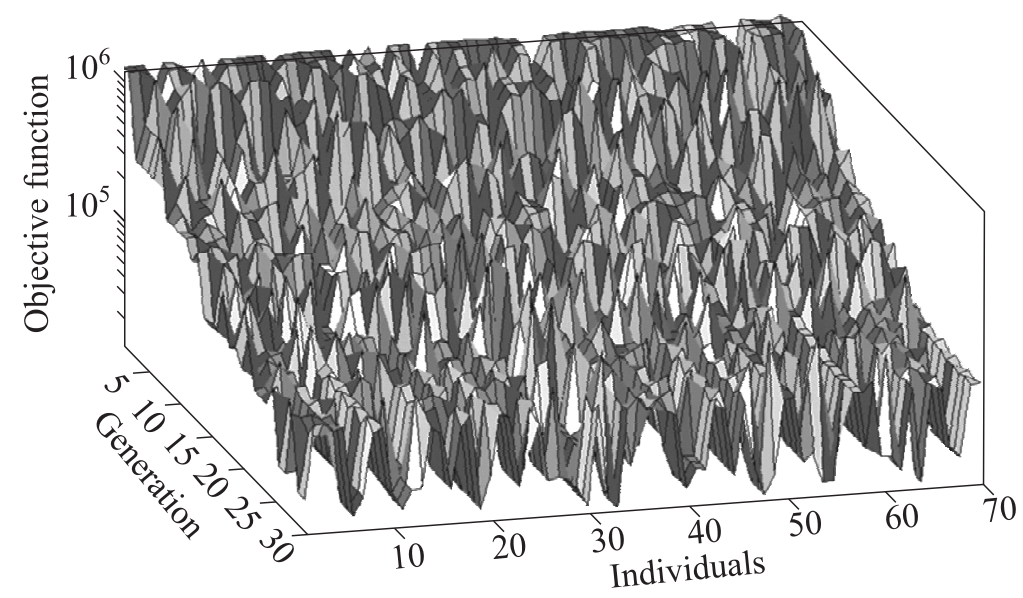

(b)

Figure 10 The GA convergence for extreme scenarii $1(a)$ and $4(b)$

(3) the ACS fuel budget;

(4) the 99.7 percent (or $3 \sigma$ value) final time-to-go (one recalls that the objective of the guidance is to null the miss-distance at $T=0 \mathrm{~s}$ ); and

(5) the failure rate expressed with respect to the number of simulated cases (1000 MTCL runs are considered) yielding a final miss-range over $50 \mathrm{~m}$ (the 25-meter hit probability is only a secondary objective not retained at this level of comparison). 

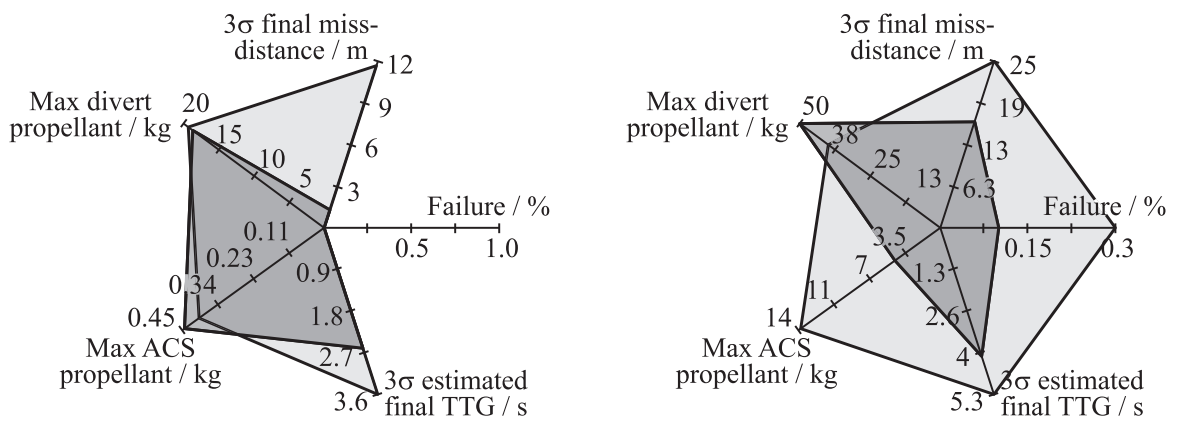

(a)
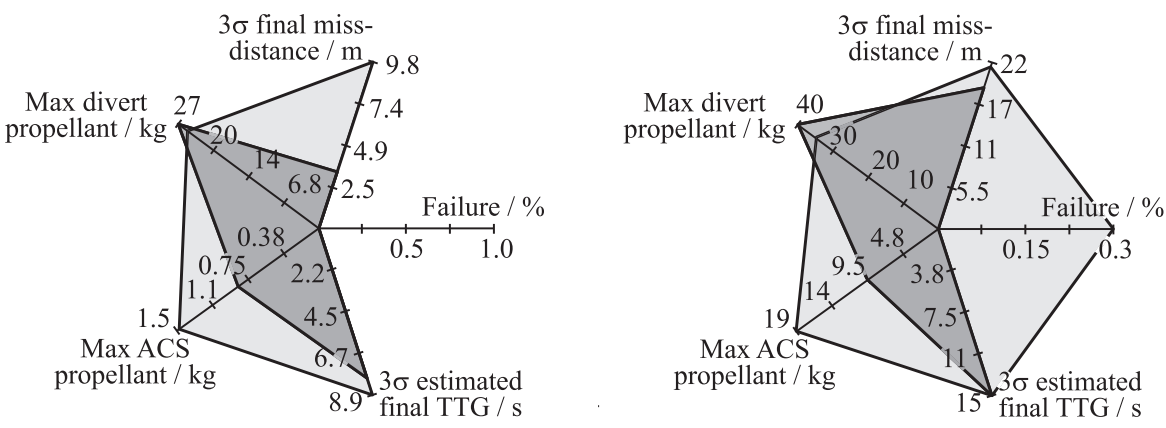

(b)
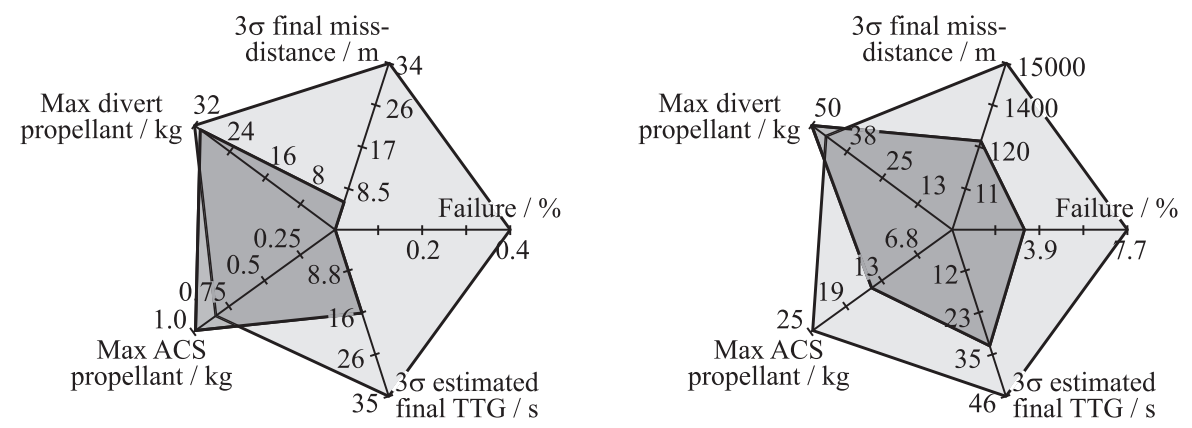

(c)

Figure 11 Handmade vs GA GNC settings: (a) 30-kilometer initial miss-distance; (b) 90-; (c) 200-kilometer initial miss-distance; left column - 200-newton divert; and right column - 3500-newton divert 
Table 7 Monte-Carlo results with GA optimization

\begin{tabular}{clcccc}
\hline \multirow{2}{*}{ Scenario } & Setting & \multicolumn{2}{c}{ 200-newton divert } & \multicolumn{2}{c}{3500 -newton divert } \\
\cline { 3 - 6 } & method & Phit $_{50 \mathrm{~m}}$ & Phit $_{25 \mathrm{~m}}$ & Phit $_{50 \mathrm{~m}}$ & Phit $_{25 \mathrm{~m}}$ \\
\hline \multirow{2}{*}{$1(30 \mathrm{~km}, 3 \sigma)$} & Handmade & $100 \%$ & $100 \%$ & $99.8 \%$ & $99.7 \%$ \\
& GA & $100 \%$ & $100 \%$ & $99.9 \%$ & $99.9 \%$ \\
\hline \multirow{2}{*}{$2(90 \mathrm{~km}, 3 \sigma)$} & Handmade & $100 \%$ & $100 \%$ & $99.8 \%$ & $99.7 \%$ \\
& GA & $100 \%$ & $100 \%$ & $100 \%$ & $100 \%$ \\
\hline \multirow{2}{*}{$3(200 \mathrm{~km}, 3 \sigma)$} & Handmade & $99.9 \%$ & $99.6 \%$ & $97.5 \%$ & $92.3 \%$ \\
& GA & $100 \%$ & $100 \%$ & $98.8 \%$ & $96.8 \%$ \\
\hline \multirow{2}{*}{$4(600 \mathrm{~km}, 3 \sigma)$} & Handmade & $99.9 \%$ & $99.3 \%$ & $86.1 \%$ & $67.0 \%$ \\
& GA & NA & NA & NA & NA \\
\hline
\end{tabular}

It is clear here that the GA process is able to optimize the GNC performance acting on the GNC data set even if in some cases, the maximum divert consumption may be larger than with the handmade tuning. But if the 50- or 25-meter hit probabilities obtained on the final 1000 runs MTCL runs are analyzed, one gets the results summarized in Table 7.

In all the cases, the GA-based settings process allows a better GNC performance. Nevertheless, the gains remain limited with respect to the 50- or 25-meter hit probabilities, but the extensive and time-consuming preliminary handmade guidance and control settings yielded already interesting results.

At this level of the GNC optimization, a specific GNC data set has been defined for each scenario. Ideally, we would rely in the end on a single GNC data set enabling the fulfilment of the mission requirement for any kind of endgame conditions. However, because most of these GNC tuning parameters are scenarioor time-dependent, it is clearly not sure to perform such task with the same level of GNC performance for very different endgame scenarios unless entering the endgame phase with the same predefined timing (the one of the longest scenarios) for any kind of initial conditions.

\section{COUPLED GNC SETTINGS AND DACS DESIGN OPTIMIZATION}

One of the baselines of the NEOShield program was to consider at least existing propulsive solutions for the DACS, hence the choice for the 10-, 200-, and 3500-newton divert options. Nevertheless, such choice was maybe not the most adequate, the analysis of some failure cases highlighting a DACS definition issue, for instance, underestimation of the ACS needs for the 3500-newton divert and a 20-hertz image-based relative navigation. Thus, the driving idea here is to add the main features of the DACS, i. e., thrust level and firing period (still considering a pulsed-mode operating DACS) to the set of GNC data to be optimized 

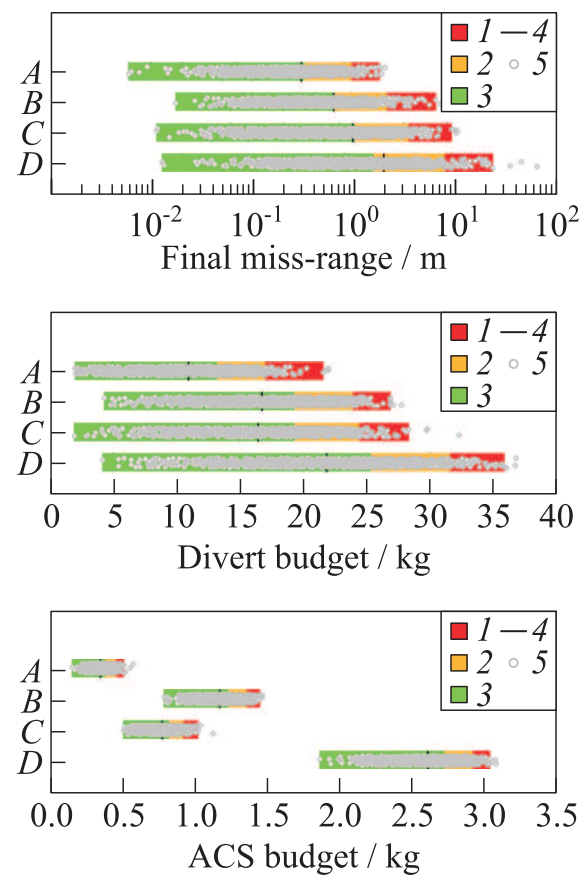

Figure 12 The GNC data and DACS optimization: $A-$ scenario $1,30 \mathrm{~km} ; B-$ scenario $2,90 \mathrm{~km} ; C$ - scenario $3,200 \mathrm{~km} ; D$ - scenario $4,600 \mathrm{~km}$; left column 200-newton divert; right column - 3500-newton divert; $1-99.7 \%$ or $3 \sigma$ domain; $2-95.4 \%$ or $2 \sigma$ domain; $3-68.2 \%$ or $1 \sigma$ domain; 4 - arithmetic mean value; and 5 - MTCL runs

by the GA process. In the end, the number of genes of an individual is raised to 20 ( 2 for the divert and ACS firing periods and 3 for the divert, yaw-pitch, and roll ACS thrust levels). The variation range of the divert thrust has been set to $[100 \mathrm{~N} ; 5000 \mathrm{~N}$ ], for a minimum command period of $50 \mathrm{~ms}$ for the Control function, compliant with the 20-hertz image-based navigation.

Without modifying the main settings of the GA processes (the optimization remains scenario-dependent), one gets the following results concerning the final miss-range and the DACS fuel budgets (Fig. 12).

Before detailing the optimization results, the first point to highlight is that the GA process has converged on the scenario 4 that was not the case when only the GNC data had to be optimized. That convergence is mainly due to the additional propulsive data whose GA setting enables a better adequation between the GNC functions, the DACS definition as previously considered being in the end too stressing for the endgame conditions of scenario 4 .

The results of the augmented GA process are summarized in Table 8. 
Table 8 Monte-Carlo results with coupled GNC and DACS GA optimization

\begin{tabular}{|c|c|c|c|c|c|c|c|c|c|}
\hline \multirow{2}{*}{$\begin{array}{l}\text { Sce- } \\
\text { nario }\end{array}$} & \multirow{2}{*}{$\begin{array}{c}\text { Divert } \\
\text { thrust, } \\
\mathrm{N}\end{array}$} & \multicolumn{2}{|c|}{ ACS thrust, $\mathrm{N}$} & \multirow{2}{*}{$\begin{array}{c}\text { Guidance } \\
\text { loop, } \\
\text { ms }\end{array}$} & \multirow{2}{*}{$\begin{array}{c}\text { Control } \\
\text { loop, } \\
\text { ms }\end{array}$} & \multicolumn{2}{|c|}{$\begin{array}{l}\text { Budget } \\
(\max ), \mathrm{kg}\end{array}$} & \multicolumn{2}{|c|}{ Hit probability, $\%$} \\
\hline & & $\begin{array}{c}\text { Pitch } \\
\text { and yaw }\end{array}$ & Roll & & & Divert & ACS & 25-meter & 50-meter \\
\hline 1 & 230 & 9 & 11 & 500 & 100 & 22 & 0.6 & 100 & 100 \\
\hline 2 & 483 & 23 & 42 & 500 & 100 & 28 & 1.5 & 100 & 100 \\
\hline 3 & 440 & 20 & 24 & 150 & 50 & 32 & 1.2 & 100 & 100 \\
\hline 4 & 289 & 15 & 46 & 150 & 100 & 37 & 3.1 & 99.7 & 99.9 \\
\hline
\end{tabular}

According to these results, there is no real need for a high thrust level divert, the obtained thrust levels ranging from roughly 200 to 500 N. For an extended endgame duration and an enlarged variation range of the divert thrust level, a lower divert thrust level would probably have been selected by the GA process. But there is also no unique DACS solution and it is not possible to define a clear trend in the sizing of the DACS or to derive sizing rules from the obtained results. In addition, the GA sizing of the DACS provides results that are different from the ones that would have been obtained using in-house explicit sizing methods. These differences may be explained by the fact that those sizing principles rely on some simplification that may be oversizing in some cases and using a stochastic method as used by a GA process enables a relaxation of the DACS sizing. In addition, divert and ACS fuel budgets are reduced. If it is quite direct to derive a relation between the endgame conditions (maximum miss-distance and timeto-go at endgame handover), the divert fuel budget and the divert thrust, the ACS consumption which is mainly linked to the duration of the endgame phase does not evolve as expected: scenarios 2 and 3 have similar ACS values, but the maximum fuel consumption for the scenario 2, which is twice shorter than scenario 3 , is $30 \%$ higher. This overconsumption that was already observed when only the GNC data were optimized is essentially the result of a different GA sizing of the camera tracking zone but the fuel budget remains limited.

Last but not least because this is the most important result with respect to the mission objective, the better adaptation of the DACS to the GNC data enables an important improvement of the GNC performance with 25- or 50-meter hit probability equal or very close to $100 \%$. This higher performance of the GNC demonstrates the interest of relying on a joint optimization of the GNC data set and the main DACS features.

\section{CONCLUDING REMARKS}

Among the different mitigations techniques investigated within the frame of the NEOShield program funded by the European Commission (FP7 framework), the 
kinetic impactor appeared the most promising to deviate a threatening asteroid far away from the Earth. The analysis performed during the NEOShield activities demonstrated that a kinetic impactor with a medium $(200 \mathrm{~N})$ to high $(3500 \mathrm{~N})$ thrust liquid-fuelled divert or even a very low thrust level $(10 \mathrm{~N})$ for a satellite-like KI was able to impact an asteroid with a final miss-range below $50 \mathrm{~m}$, or even $25 \mathrm{~m}$, in most of the cases, even for very degraded conditions at endgame handover. Nevertheless, such performance level that was established for a given definition of the divert and ACS (driving idea being to consider existing propulsive system) left some room for improvement of the GNC performance, only time-consuming handmade settings of the GNC data set having been done.

Limiting the analysis to the medium to high thrust divert KI and to each endgame scenario (from very optimistic to very pessimistic conditions at endgame handover), it was then decided to replace the classic handmade setting of the GNC data set (15 parameters to tune) by an optimization process relying on GA. As usual with such optimization method, the most challenging aspect is the definition of the objective function and the GA parameters (number of chromosomes, individuals, epochs, mutation, reinsertion, selection, etc.). But once this preliminary step is completed after some iterations, the optimization allows an improvement of the GNC performance in addition of shortening the setting phase. Discarding the possible reuse of existing DACS solution that may be undersized for some degraded endgame conditions and considering 5 additional genes, it is possible to perform a coupled GNC-DACS optimization which yields a much larger improvement of the performance.

According to these results, next step would be to rerun the GA optimization process restricted to the GNC data set and considering an intermediate and unique DACS definition (for instance, the one obtained with the GNC-DACS optimization on the worst endgame scenario) and to redefine the set of the GNC data to remove the time-dependency of some parameters in order to enable a unique GNC setting compliant with all the retained endgame scenarii.

\section{REFERENCES}

1. Chapuy, M., P. Vernis, N. Desprée, and F. Capolupo. 2014. NEOShield Project: GNC design and performance assessment of a liquid-fuelled asteroid kinetic impactor. 9th Conference (International) on Guidance, Navigation, and Control Systems. Porto, Portugal.

2. Vernis, P., J.-F. Jourdas, N. Hachelef, C. Gu, and A. Scwhientek. 2013. HOMER project: ILIAD and ODYSSEY GNC design and performance. 4th International ARA Days. Arcachon, France.

3. Murtaugh, S. A., and H.E. Criel. 1966. Fundamentals of proportional navigation. IEEE Spectrum 3:75-85. 
4. Pfleghaar, W. 1983. The general class of optimal proportional navigation. J. Guid. Control Dynam. 8(1):144-147.

5. Shukla, U.S., and P.R. Mahapatra. 1989. Efficient atmospheric and extraatmospheric interception through optimally biased proportional navigation. IEEE National Aerospace and Electronics Conference Proceedings. New York, NY: IEEE. $156-166$.

6. Ben Asher, J.Z. 1994. Linear-quadratic pursuit-evasion games with terminal velocity constraints. AIAA Paper No. 94-3567-CP.

7. Shima, T., and J. Shinar. 2002. Time-varying linear pursuit-evasion game models with bounded controls. J. Guid. Control Dynam. 25(3):425-432.

8. Hawkins, M., Y. Guo, and B. Wie. 2011. Guidance algorithms for asteroid intercept missions with precision targeting requirements. AAS Paper No. 11-531.

9. Hawkins, M., and B. Wie. 2012. Impact-angle control of asteroid interceptors/penetrators. AAS Paper No. 11-271.

10. Stallard, D. V. 1987. An angle-only tracking filter in modified spherical coordinates. AIAA Guidance, Navigation and Control Conference Proceedings. Monterey, CA: AIAA. 542-550.

11. Vernis, P., V. Morio, and E. Ferreira. 2007. Genetic algorithms for coupled RLV trajectory and guidance optimization. 17th IFAC Symposium on Automatic Control in Aerospace Proceedings. Toulouse, France. 